\title{
Inhibition of Autophagy Induction Delays Neuronal Cell Loss Caused by Dysfunctional ESCRT-III in Frontotemporal Dementia
}

\author{
Jin-A Lee and Fen-Biao Gao \\ Gladstone Institute of Neurological Disease and Department of Neurology, University of California, San Francisco, San Francisco, California 94158
}

\begin{abstract}
Autophagy is a conserved lysosomal protein degradation pathway whose precise roles in age-dependent neurodegenerative diseases remain largely unknown. Here we show that the autophagy inhibitor 3-methyladenine delays neuronal cell loss caused by dysfunctional endosomal sorting complex required for transport III (ESCRT-III), either through loss of its essential component mSnf7-2 or ectopic expression of the disease protein CHMP2B ${ }^{\text {Intron5 }}$, which is associated with frontotemporal dementia linked to chromosome 3 . Neuronal loss was also delayed by reduced activity of the autophagy genes $\operatorname{atg} 5$ and $\operatorname{atg} 7$. However, the endosomal accumulation of ubiquitinated proteins induced by dysfunctional ESCRT-III was not significantly affected, further confirming the essential contribution of dysregulated autophagy pathway in neurodegeneration. These findings show that autophagic stress by excess accumulation of autophagosomes is detrimental to neuronal survival under certain neurodegenerative conditions.
\end{abstract}

\section{Introduction}

Frontotemporal dementia (FTD), the most common form of age-dependent neurodegeneration before the age of 60 , is associated with focal atrophy of the frontal and/or temporal lobes (Vossel and Miller, 2008). Several proteins have been implicated in FTD pathogenesis, such as Tau (Hong et al., 1998; Hutton et al., 1998), valosin-containing protein (Watts et al., 2004), CHMP2B (Skibinski et al., 2005), progranulin (Baker et al., 2006; Cruts et al., 2006), and TDP-43 (Neumann et al., 2006). CHMP2B is a component of the endosomal sorting complex required for transport III (ESCRT-III) whose rare mutation was found in a large Danish FTD3 family (Skibinski et al., 2005). ESCRT-III is required for trafficking of ubiquitinated transmembrane proteins from early endosomes to luminal vesicles in multivesicular bodies and other biological processes (Hurley, 2008). How mutant CHMP2B causes neurodegeneration remains incompletely understood.

Although CHMP2B mutations are rare in FTD patient populations, the mutant protein $\mathrm{CHMP} 2 \mathrm{~B}^{\text {Intron5 }}$ causes dendritic retraction, autophagosome accumulation, and neuronal cell loss in cultured mature cortical neurons (Lee et al., 2007). These neurotoxic effects reflect the failure of $\mathrm{CHMP} 2 \mathrm{~B}^{\text {Intron5 }}$ to dissociate from ESCRT-III-containing mSnf7-2 (Lee et al., 2007). Both the proper assembly and disassembly are required

Received Feb. 23, 2009; revised May 12, 2009; accepted May 26, 2009.

This work was supported by the California Institute of Regenerative Medicine (J.L.) and by the National Institutes of Health (NIH) (F.-B.G.) and NIH Grant C06 RR018928 (to the J. David Gladstone Institutes). We thank N. Mizushima for atg 5 knock-out mice, L. Liu for assistance with the mice genotyping and cortical cultures, J. Wong for help with electron microscopy, and S. Ordway and G. Howard for editorial assistance.

Correspondence should be addressed to Fen-Biao Gao, Gladstone Institute of Neurological Disease, 1650 Owens Street, San Francisco, CA 94158. E-mail: fgao@gladstone.ucsf.edu.

DOl:10.1523/JNEUROSCI.0924-09.2009

Copyright $\odot 2009$ Society for Neuroscience $\quad$ 0270-6474/09/298506-06\$15.00/0 for the normal function of ESCRT-III (Hurley, 2008). Indeed, small interfering RNA (siRNA) knockdown of mSnf7-2 or expression of a dominant-negative form of SKD1, an AAAtype ATPase essential for ESCRT-III disassembly, also cause autophagosome accumulation and neuronal cell loss (Lee et al., 2007). Loss of other ESCRT components also disrupts the autophagy pathway in non-neuronal mammalian cells (Filimonenko et al., 2007).

Autophagy (macroautophagy) is a highly conserved bulk degradation pathway that is important for differentiation, homeostasis, and survival under physiological and pathophysiological conditions (Mizushima et al., 2008). Cytosolic proteins and organelles are sequestered by a double membrane mostly in a nonspecific manner. The resulting vacuoles, called autophagosomes, go through a series of maturation steps and eventually fuse with lysosomes for degradation. The autophagy pathway is associated with many neurodegenerative diseases. For instance, neuronal autophagy is activated early in Alzheimer's disease and becomes abnormal as the disease progresses (Boland et al., 2008). $\alpha$-Synuclein mutants implicated in Parkinson's disease block their own degradation by the chaperone-mediated autophagy pathway, leading to a gain-offunction toxicity (Cuervo et al., 2004). Moreover, activated autophagy helps the clearance of toxic polyglutaminecontaining proteins in Huntington's disease (Ravikumar et al., 2004). Although it is generally believed that autophagy is beneficial to neuronal survival, its precise roles in age-dependent neurodegenerative diseases remain unclear. In particular, it is unclear whether autophagosome accumulation is protective or detrimental to neuronal survival at different pathogenic stages of FTD3 or other neurodegenerative diseases involving defects in the endosomal-lysosomal pathway. This important issue is partially addressed in the study below. 

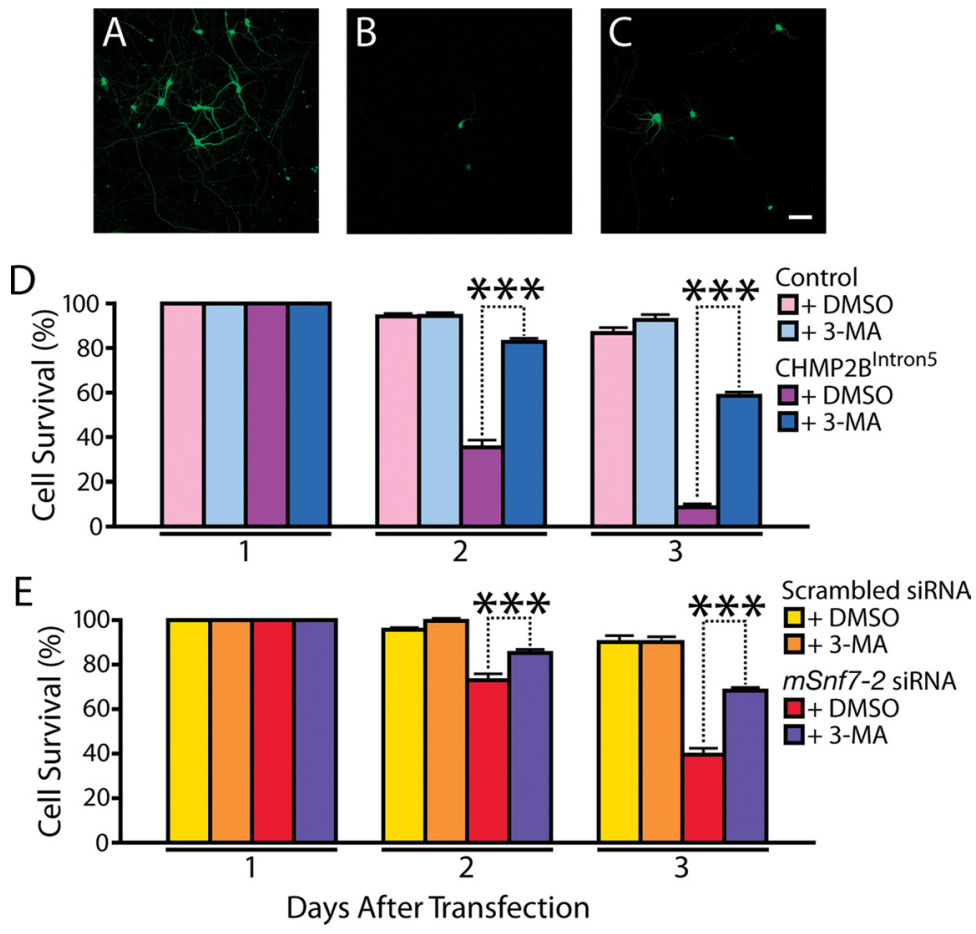

Figure 1. The autophagy inhibitor 3-MA partially suppresses neuronal cell loss caused by dysfunctional ESCRT-III. A, An image of cultured rat cortical neurons $3 \mathrm{~d}$ after transfection with $\mathrm{CHMP}_{2 \mathrm{~B}}{ }^{\mathrm{WT}}$ and treated with DMSO. $\boldsymbol{B}$, An image of mature cortical neurons $3 \mathrm{~d}$ after transfection with the FTD3-associated mutant protein CHMP2B ${ }^{\text {Intron5 }}$ showing extensive neuronal cell loss. In this experiment, DMSO was added as the control for C. C, 3-MA in DMSO was added to the cultured medium, which increased the survival of cortical neurons expressing CHMP2B $^{\text {Intron5 }}$. In $A-C$, the green signals are from GFP cotransfected with different forms of CHMP2B. D, Survival of mature cortical neurons expressing the FTD3-associated mutant protein CHMP2B ${ }^{\ln t r o n 5}$ in the presence or absence of 3-MA. $\boldsymbol{E}$, Survival of mature cortical neurons with reduced mSnf7-2 activity in the presence or absence of 3-MA. Values are mean \pm SEM of three or four independent experiments. ${ }^{* * *} p<0.001$ as determined by ANOVA with Newman-Keuls multiple test. Because of space limitation, only the values for 1, 2, and $3 \mathrm{~d}$ after transfection are presented in this figure. Scale bar: (in $\mathbf{C}$ A-C, $50 \mu \mathrm{m}$.

\section{Materials and Methods}

Generation and validation of $\operatorname{atg} 5$ and $\operatorname{atg} 7$ siRNAs. To generate genespecific siRNA constructs, two oligonucleotides were annealed and cloned into the pSUPER-GFP vector (Oligoengine). The target sequences are listed in supplemental Table S1 (available at www. jneurosci.org as supplemental material). For lentivirus generation, atg5, atg7, or scrambled siRNAs, including the $\mathrm{H} 1$ promoter, were cloned into the FUGW vector. For the validation of each siRNA, mature cortical neurons were infected with lentivirus containing atg5 or atg7 siRNAs. Western blot was performed using Atg5 or Atg7 antibody (1:1000; Novus Biological) $4-5 \mathrm{~d}$ after infection.

Culture and treatment of primary cortical neurons. Primary cortical neurons were isolated from embryonic day 18 (E18) Sprague Dawley rats (Charles River) and $\operatorname{atg} 5^{-/-}$mice. The absence of Atg 5 was confirmed by genomic PCR and Western blot with Atg5 antibody (1: 1000; Novus Biological). Neurons were transfected with Lipofectamine 2000 (Invitrogen) or infected with lentivirus at $15 \mathrm{~d}$ in vitro. Transfected neurons always express green fluorescent protein (GFP) and the number of GFP-positive neurons at $1 \mathrm{~d}$ after transfection was used as the baseline for quantification of neuronal survival at later days. Neuronal viability was assessed with a propidium iodide (PI) exclusion assay. GFP-positive and PI-negative neurons were counted as surviving neurons $1-3 \mathrm{~d}$ after transfection. All experiments were repeated three or four times. For inhibition of autophagy induction, mature cortical neurons transfected with $\mathrm{CHMP}^{2} \mathrm{~B}^{\text {Intron5 }}$ or $m S n f 7-2$ siRNA were treated with $5 \mathrm{~mm}$ 3-methyladenine (3-MA) (Sigma) or $10 \mu \mathrm{M}$ wortmannin (Sigma). For inhibition of autophagy with siRNAs, cells were transfected with $m S n f 7-2$ siRNA or CHMP2B ${ }^{\text {Intron5 }} 3-5 \mathrm{~d}$ after each viral infection.
For all neuronal cell survival analysis, ANOVA with Newman-Keuls multiple test (post hoc comparison) was performed to compare the statistical significance between groups. For localization of LC3 or Rab7, GFP-LC3 or GFP-Rab7 was cotransfected with CHMP2B ${ }^{\text {Intron } 5}$ or $m S n f 7-2$ siRNA.

Western blot and immunostaining. Neurons were homogenized in radioimmunoprecipitation assay buffer ( $50 \mathrm{~mm}$ Tris- $\mathrm{HCl}, \mathrm{pH} 7.5$, $150 \mathrm{~mm} \mathrm{NaCl}, 0.1 \% \mathrm{NP}-40,0.1 \%$ SDS, and $0.5 \%$ sodium deoxycholate). The samples were analyzed by Western blot with anti-LC3 (1:1000), anti-ubiquitin (1:1000), anti$m S n f 7-2$ (1:1000), or anti-actin (1:2000) and horseradish peroxidase-conjugated antirabbit or anti-mouse secondary antibody (1: 4000). Immunostaining experiments were performed as described previously (Lee et al., 2007).

Electron microscopy. Cultured cortical neurons from wild-type (WT) or atg $5^{-/-}$mice were infected with $m S n f 7-2$ siRNA and fixed in $2 \%$ glutaraldehyde, $1 \%$ paraformaldehyde, and $100 \mathrm{~mm}$ sodium cacodylate, $\mathrm{pH}$ 7.4. For induction of starvation, cultured mature cortical neurons were incubated in Earle's balanced salt solution (Invitrogen) for $24 \mathrm{~h}$. For electron microscopy of $m S n f 7-2$ knock-out mice, embryos were collected at E6.5 and fixed. Tissue was then postfixed in $2 \%$ osmium tetroxide, block stained in $2 \%$ aqueous uranyl acetate, dehydrated in acetone, and embedded in LX-112 resin (Ladd Research Industries). Ultrathin sections were contrast stained with $0.8 \%$ lead citrate, examined on a Jeol JEM-1230 electron microscope, and photographed with a Gatan Ultrascan USC1000 digital camera.

\section{Results}

An autophagy inhibitor delays dysfunctional ESCRT-IIIinduced neuronal cell loss

Previous studies indicate that dysfunctional ESCRT-III causes autophagosome accumulation, which is probably attributable to the blockage of autophagosome maturation (Filimonenko et al., 2007; Lee et al., 2007; Lee and Gao, 2008). To assess whether autophagosome accumulation is beneficial or detrimental to neuronal survival in the presence of mutant CHMP2B associated with FTD3, we isolated cortical neurons from E18 embryos, cultured them for $15 \mathrm{~d}$, and transfected the well differentiated mature neurons with a CHMP2B ${ }^{\text {WT }}$ (Fig. $1 A$ ) or CHMP2B ${ }^{\text {Intron5 }}$ construct (Fig. $1 B, C$ ) together with a GFP construct. We treated mature cortical neurons with the autophagy inhibitor 3-MA, which suppresses the activity of Vps34, a class III phosphoinositide-3 (PI3) kinase that interacts with Beclin 1 in vesicle nucleation during autophagy induction (Seglen and Gordon, 1982; Mizushima et al., 2008). Treatment with 5 mM 3-MA did not completely block but instead delayed neuronal cell loss caused by $C H M P 2 B^{\text {Intron5 }}$ expression (Fig. $1 C, D$ ). For instance, $3 \mathrm{~d}$ after transfection, $58.4 \%$ of CHMP2B ${ }^{\text {Intron5 }}$-expressing neurons in the presence of 3-MA, but only $9.2 \%$ in the absence of 3 -MA, were viable $(p<0.001)$ (Fig. $1 D)$. As a negative control, 3-MA treatment itself did not affect neuronal survival (Fig. 1D). To further confirm the effect of autophagy inhibition on neuronal cell survival, we treated the cells with wortmannin, another 
small molecule that inhibits Vps34 and other PI3 kinases (Blommaart et al., 1997). This treatment also did not completely suppress but indeed slowed down CHMP2 ${ }^{\text {Intron5 }}$-induced neuronal cell loss (supplemental Fig. S1, available at www. jneurosci.org as supplemental material).

Our previous work indicated that siRNA knockdown of mSnf7-2 but not CHMP2B activity causes autophagosome accumulation and neuronal cell death and that CHMP2B ${ }^{\text {Intron5 }}$ neurotoxicity is mediated through its stronger association with mSnf7-2 (Lee et al., 2007). Thus, we also examined the effect of $5 \mathrm{~mm}$ 3-MA on neuronal cell loss caused by reduced mSnf7-2 activity. We found that, 2 or $3 \mathrm{~d}$ after siRNA knockdown of mSnf7-2, the neuronal survival rate was higher in the presence of 3-MA than that in the absence of 3-MA (Fig. $1 E)$. The effects of 3-MA at 4 or $5 \mathrm{~d}$ after transfection with $m S n f 7-2$ siRNA or CHMP2B ${ }^{\text {Intron5 }}$ were also evident (data not shown). To confirm that 3-MA indeed suppressed the autophagy pathway in neurons with dysfunctional ESCRTIII, we found that 3-MA inhibited the increases in the level of endogenous LC3-II and LC-II/LC-I ratio (supplemental Fig. S2, available at www. jneurosci.org as supplemental material), which are thought to be biochemical indicators of autophagosome formation (Klionsky et al., 2008). The toxicity of CHMP2B precluded Western blot analysis because CHMP2B ${ }^{\text {Intron5 }}$-containing lentiviral particles from HEK-FT cells could not be generated. Together, these pharmacological analyses raise the possibility that autophagosome accumulation attributable to blockage of their maturation contributes to neuronal cell loss caused by mutant CHMP2B associated with FTD3.

\section{Inhibition of autophagy gene function delays neuronal cell loss induced by dysfunctional ESCRT-III}

Because 3-MA and wortmannin inhibit some PI3 kinases not specifically involved in the autophagy pathway, we wanted to confirm that the pharmacological rescue of neuronal cell death truly reflected inhibition of autophagy induction. We suppressed the expression of autophagy-specific genes and assessed the effects on neuronal cell loss induced by dysfunctional ESCRT-III. We first tested Atg7, an E1-like enzyme required to activate Atg12 and Atg8 (LC3) during the initial steps of autophagosome formation (Mizushima et al., 1998). One of several siRNA constructs specific to different regions of atg7 mRNA (Fig. 2A) decreased the level of the endogenous LC3-II and the LC-II/LC-I ratio from 0.9 in $m S n f 7-2$ siRNAtransfected neurons to 0.4 in $m S n f 7-2$ siRNA and $\operatorname{atg} 7$ siRNAtransfected neurons (Fig. $2 B$ ), consistent with reduced autophagosome formation. Correspondingly, knockdown of Atg7 significantly improved the survival rate of mature rat cortical neurons expressing CHMP2B ${ }^{\text {Intron5 }}$ (Fig. $2 C-F$ ). For instance, $3 \mathrm{~d}$ after transfection, $57 \%$ of CHMP $2 \mathrm{~B}^{\text {Intron5 }}$-expressing neurons with reduced Atg7 activity, but only $27 \%$ with functional Atg7, were viable $(p<0.001)$. Knockdown of Atg7 also delayed neuronal cell loss in cortical neurons with reduced mSnf7-2 activity (56 vs 33\%) at day 3 after transfection $(p<$ 0.001 ) (Fig. 2G). Similarly, knockdown of Atg5 (supplemental Fig. S3A, available at www.jneurosci.org as supplemental material), which is required for the elongation of isolation membranes during autophagosome formation (Mizushima et al., 2001), also delayed neuronal cell loss caused by reduced mSnf7-2 activity (supplemental Fig. S3B-D, available at www. jneurosci.org as supplemental material) or $\mathrm{CHMP} 2 \mathrm{~B}^{\text {Intron5 }}$ expression (supplemental Fig. S $3 E-G$, available at www. jneurosci.org as supplemental material). Thus, excess accumulation of autophagosomes contributes to eventual neuronal cell loss when ESCRT-III is dysfunctional. 

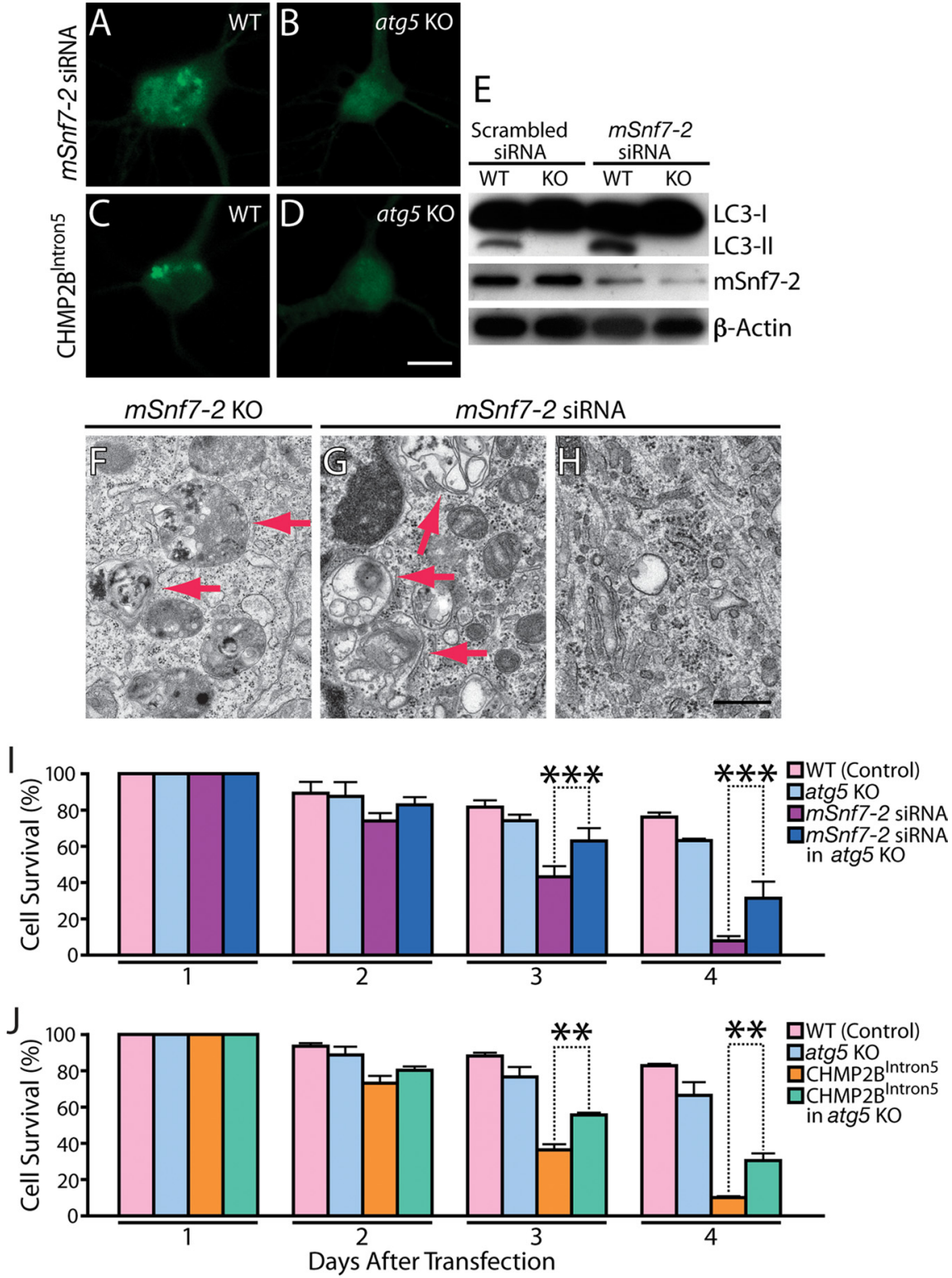

Figure 3. Atg $5^{-/-}$cortical neurons were more resistant to neuronal cell loss induced by dysfunctional ESCRT-III. A, Reduced mSnf7-2 activity causes accumulation of GFP-LC3-positive autophagosomes in wild-type cortical neurons. $\boldsymbol{B}$, Reduced mSnf7-2 activity does not cause the accumulation of GFP-LC3-positive autophagosomes in $a t g 5^{-/-}$cortical neurons. C, Expression of CHMP $2 B^{\text {Intron5 }}$ causes accumulation of GFP-LC3-positive autophagosomes in wild-type neurons. D, Expression of CHMP2B $B^{\text {Intron5 }}$ did not cause accumulation of GFP-LC3-positive autophagosomes in atg $5^{-/-}$neurons. Scale bar: (in D) $\boldsymbol{A}-\boldsymbol{D}, 10 \mu \mathrm{m}$. E, mSnf7-2 siRNA did not cause autophagosome formation in cortical neurons isolated from atg $5^{-1-}$ mouse embryos, as indicated by the absence of $\mathrm{LC} 3-I I$ on Western blot. Note that the relative level of LC3-II is much lower than in rat neurons. $F$, Electron microscopy showed many autophagosomes in $m$ Snf7-2 knock-out mice embryos (E6-E6.5). G, Accumulation of autophagosomes is evident in $\mathrm{mSnf7-2-deficient} \mathrm{cortical} \mathrm{neurons.} \boldsymbol{H}, \boldsymbol{m S n f 7 - 2}$ siRNA did not induce autophagosome accumulation in atg 5 knock-out neurons as shown by electron microscopy. Red arrows indicate autophagosomes. Scale bar: (in $\boldsymbol{H}) \boldsymbol{F}-\boldsymbol{H}, 1 \mu \mathrm{m}$. I, Effects of reduced mSnf7-2 activity on survival of wild-type and $a t g 5^{-/}$cortical neurons. J, Effects of the FTD3-associated mutant protein CHMP2B ${ }^{\operatorname{lntron} 5}$ on survival of wild-type and $a t g 5^{-1-}$ cortical neurons. Values are mean \pm SEM from three or four independent experiments for neurons 1,2 , and $3 \mathrm{~d}$ after transfection. ${ }^{* *} p<0.01,{ }^{* * *} p<0.001$ as determined by ANOVA with Newman-Keuls multiple test. KO, Knock-out.

\section{Atg5-deficient neurons are more resistant to neurotoxicity of dysfunctional ESCRT-III}

Next, we examined the neurotoxicity of CHMP2 $\mathrm{B}^{\text {Intron5 }}$ in cortical neurons isolated from atg5 knock-out $\left(\operatorname{atg} 5^{-/-}\right)$mice (Kuma et al., 2004). Although these mice die during the neonatal starvation period (Kuma et al., 2004), atg $5^{-/-}$postmitotic neurons survive for weeks before abnormal proteins eventually accu- mulate in these cells (Hara et al., 2006). We isolated cortical neurons from E18 atg5 knock-out mouse embryos and confirmed the absence of Atg 5 by Western blot (data not shown). These cultured neurons survive weeks without $\operatorname{Atg} 5$ as long as they are maintained in neuronal medium (B27 in Neurobasal medium) (data not shown). Although LC3-GFP-positive autophagosomes accumulate in starved wild-type cortical neurons, none were observed in starved $\operatorname{atg} 5^{-/-}$cortical neurons cultured for 15-18 d (supplemental Fig. S4, available at www.jneurosci.org as supplemental material), confirming an essential role of Atg5 in autophagosome formation in culture neurons isolated from atg 5 knock-out mouse embryos.

To further demonstrate that reduction of autophagic stress by inhibition of autophagy induction contributes to neuronal cell survival even in the presence of ubiquitinated protein aggregation, we performed the following experiments. None of the atg5 knock-out neurons showed any LC3GFP-positive autophagosomes when mSnf7-2 was knocked down (Fig. $3 B$ ) or CHMP2B ${ }^{\text {Intron5 }}$ was expressed (Fig. $3 D$ ). Correspondingly, Atg 5 deficiency abolished the increase in the expression of endogenous LC3-II caused by loss of mSnf7-2 or ectopic expression of CHMP2B ${ }^{\text {Intron5 }}$ (Fig. $3 E$ ). Electron microscopy revealed a number of autophagosomes in neurons from E7.5 mSnf7-2 knock-out embryos, suggesting that depletion of ESCRT in vivo also causes defects in the autophagy pathway (Fig. $3 F$ ). As expected, autophagosomes accumulated in mouse cortical neurons expressing mSnf7-2 siRNA (Fig. 3G) were completely absent in cortical neurons cultured from E18 atg $5^{-1-}$ embryos and infected with lentiviral mSnf7-2 siRNA (Fig. $3 H$ ).

Thus, three lines of evidence (GFPLC3, Western blot, and EM analysis) confirm that inhibition of autophagy induction by loss of atg5 activity prevents autophagosome accumulation caused by dysfunctional ESCRT-III. In agreement with the 3-MA treatment and siRNA knockdown of atg7, autophagy inhibition by loss of atg5 activity in postmitotic neurons delayed, although did not completely block, neuronal cell loss caused by loss of mSnf7-2 (Fig. 3I). At day 4 after $m S n f 7-2$ knockdown, 30\% of Atg5-deficient neurons, but only $6 \%$ of control neurons, were viable $(p<0.001)$. The survival rate of cortical neurons expressing CHMP2B ${ }^{\text {Intron5 }}$ was also improved by the complete loss of atg 5 activity ( 54 vs $35 \%$ at day 3 after transfection and 29 vs $9 \%$ at day 4 ; both $p<0.001$ ) (Fig. $3 J$ ). These results demonstrate that reduction of autophagic stress by inhibiting the accumulation of autophagosomes at the induction stage delays neuronal cell loss induced by dysfunc- 


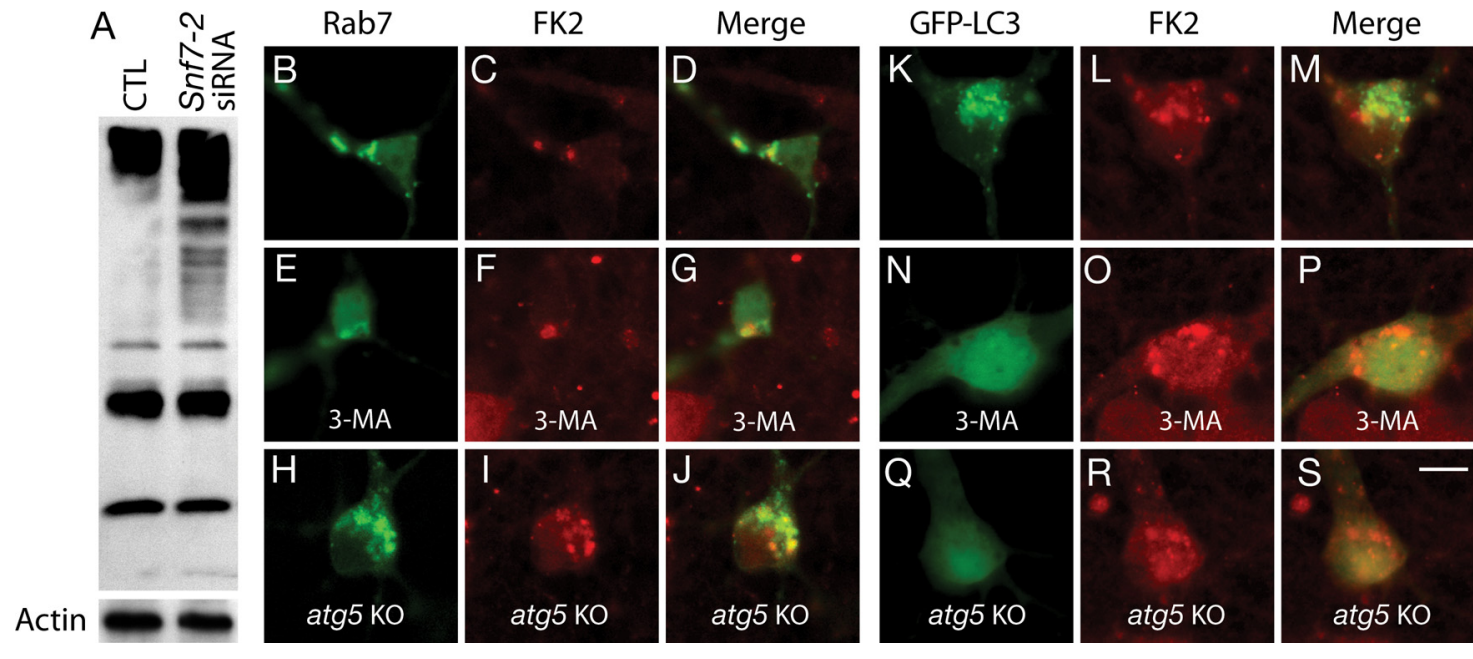

Figure 4. Uncoupling of autophagosome accumulation and ubiquitinated protein accumulation in cortical neurons. $A$, Western blot analysis of ubiquitinated proteins in mature cortical neurons $3 \mathrm{~d}$ after infection with lentiviral constructs encoding control or scrambled or mSnf7-2 siRNA. B-J, CHMP2B ${ }^{\text {Intron5 }}$ caused accumulation of FK2-positive ubiquitinated proteins in Rab7-positive endosomes $(\boldsymbol{B}-\boldsymbol{D})$, which was not qualitatively affected by $3-\mathrm{MA}$ treatment $(\boldsymbol{E}-\boldsymbol{G})$ or loss of Atg 5 activity $(\boldsymbol{H}-\boldsymbol{J})$. The neuron in $\boldsymbol{H}$ appeared to have more aggregates, probably because of its bigger size. $\boldsymbol{K}-\boldsymbol{S}, 3-M A$ treatment or loss of Atg5 activity suppressed the formation of GFP-LC3-positive autophagosomes $(\boldsymbol{N}, \mathbf{Q})$ but not the accumulation of FK2-positive ubiquitinated proteins $(\boldsymbol{O}, \boldsymbol{R})$ in cortical neurons expressing CHMP2B ${ }^{\operatorname{Intron5}}$ at day 3 . In $\boldsymbol{B}, \boldsymbol{E}$, and $\boldsymbol{H}$, the cyanine 2-conjugated secondary antibody was used. In $\boldsymbol{L}, \mathbf{O}$, and $\boldsymbol{R}$, cyanine 3-conjugated secondary antibody was used. 3-MA suppressed the appearance of GFP-LC3-positive autophagosomes in $80 \%$ CHMP2B ${ }^{\text {Intron5 }}$-expressing neurons $(\boldsymbol{N})$, whereas none of the atg 5 knock-out neurons had autophagosomes. In $\boldsymbol{K}, \boldsymbol{N}$, and $\mathbf{Q}$, GFP signals were directly collected by confocal microscopy. Scale bar: (in S) $\boldsymbol{B}-\mathbf{S}, 10 \mu \mathrm{m}$. CTL, Control; K0, knock-out.

tional ESCRT-III, indicating a detrimental role for autophagy in some neurodegenerative conditions.

\section{Uncoupling the accumulations of autophagosomes and ubiquitinated proteins in cortical neurons}

Accumulation of ubiquitinated proteins is a hallmark of many age-dependent neurodegenerative diseases. To determine whether manipulation of autophagy affects the state of ubiquitinated proteins associated with FTD3, we examined the temporal relationship between endosomal defects caused by dysfunctional ESCRT-III and autophagosome accumulation. siRNA knockdown of mSnf7-2 activity led to the accumulation of ubiquitinated proteins $3 \mathrm{~d}$ after transfection as shown by Western blot with FK2 antibody (Fig. 4A). This accumulation occurred shortly after $m S n f 7-2$ knockdown or CHMP2B ${ }^{\text {Intron5 }}$ expression, which precedes the formation of LC3-positive autophagosomes (Lee et al., 2007). In mature cortical neurons, CHMP2B ${ }^{\text {Intron5 }}$ induced the accumulation of ubiquitinated proteins that colocalized with Rab7-positive endosomes (Fig. 4B-D). The autophagy inhibitor 3-MA (Fig. 4E-G) or loss of Atg5 activity (Fig. $4 H-J$ ) did not significantly affect the number of neurons containing ubiquitinated protein aggregates. In contrast, 3-MA treatment or loss of Atg 5 suppressed the formation of GFP-LC3-positive autophagosomes in CHMP2B ${ }^{\text {Intron5}}$-expressing cortical neurons (Fig. $4 K, N, Q$ ) without affecting the formation of FK2-positive ubiquitinated protein aggregates $3 \mathrm{~d}$ after transfection (Fig. $4 M, P, S$ ). Similar findings were obtained when mSnf7-2 activity was reduced by siRNA, another way to disrupt the normal function of ESCRT-III (data not shown). Thus, neuronal cell loss caused by autophagosome accumulation and other mechanisms such as the accumulation of ubiquitinated protein aggregates can be uncoupled.

\section{Discussion}

In this study, multiple lines of evidence from pharmacological, cellular, and genetic analyses demonstrated that excess autophagosome accumulation contributes to neuronal cell loss when ESCRT-III is dysfunctional, in addition to other potential mech- anisms such as accumulation of ubiquitinated protein aggregates. Although dysfunctional ESCRT-III caused the blockage of autophagosome maturation, concurrent inhibition of autophagy induction reduced the detrimental accumulation of autophagosomes. Throughout this study, we used two complementary approaches to disrupt the normal functions of ESCRT-III. One was an siRNA-based loss-of-function approach to knockdown expression of endogenous mSnf7-2, a key component of ESCRTIII essential for animal development and neuronal survival (Lee et al., 2007). Loss of mSnf7-2 prevents the proper assembly of ESCRT-III onto endosomal membranes. We also used a gain-offunction approach. We expressed the FTD3-associated disease protein CHMP2B ${ }^{\text {Intron5 }}$, which forms a complex with $\mathrm{mSnf7-2}$ more avidly than CHMP2B ${ }^{\mathrm{WT}}$ (Lee et al., 2007), therefore disrupting the normal disassembly of ESCRT-III from endosomal membranes. Both approaches indicate that the effect of inhibition of autophagy suppression on neuronal survival is not simply attributable to the overexpression of a mutant protein.

In recent years, several disease genes have been associated with FTD (Hong et al., 1998; Hutton et al., 1998; Watts et al., 2004; Skibinski et al., 2005; Baker et al., 2006; Cruts et al., 2006; Neumann et al., 2006). However, the detailed mechanisms underlying the molecular pathogenesis of different forms of FTD remain essentially unexplored. Our findings have several important implications for our understanding of disease processes. First, in contrast to studies suggesting a beneficial role for autophagy in neurodegenerative diseases (Ravikumar et al., 2004), our results imply that excess accumulation of autophagosomes is detrimental to neuronal survival under certain circumstances (e.g., in neurodegeneration associated with endosomal-lysosomal defects). In the case of FTD3, mutant CHMP2B proteins seem to block the maturation of autophagosomes into autolysosomes (Filimonenko et al., 2007; Lee and Gao, 2008). Under these conditions, reduced induction of autophagy seems to be more beneficial than defective autophagy. It remains to be determined exactly how ESCRT components are involved in the autophagy pathway (Raiborg and Stenmark, 2009; Yue et al., 2009). Second, although 
basal autophagy is required for neuronal survival (Hara et al., 2006; Komatsu et al., 2006), either excessive production or reduced clearance may all cause dysregulation of autophagic processes, resulting in neurodegeneration. Finally, complete loss of normal ESCRT-III function causes endosomal defects and accumulation of ubiquitinated proteins, which may lead to neurodegeneration independent of the state of autophagy. Nonetheless, small molecules such as 3-MA that partially suppress autophagy induction could be potential therapeutic agents for early stages of some forms of FTD and possibly other neurodegenerative diseases.

\section{References}

Baker M, Mackenzie IR, Pickering-Brown SM, Gass J, Rademakers R, Lindholm C, Snowden J, Adamson J, Sadovnick AD, Rollinson S, Cannon A, Dwosh E, Neary D, Melquist S, Richardson A, Dickson D, Berger Z, Eriksen J, Robinson T, Zehr C, et al. (2006) Mutations in progranulin cause tau-negative frontotemporal dementia linked to chromosome 17. Nature 442:916-919.

Blommaart EF, Krause U, Schellens JP, Vreeling-Sindelárová H, Meijer AJ (1997) The phosphatidylinositol 3-kinase inhibitors wortmannin and LY294002 inhibit autophagy in isolated rat hepatocytes. Eur J Biochem 243:240-246.

Boland B, Kumar A, Lee S, Platt FM, Wegiel J, Yu WH, Nixon RA (2008) Autophagy induction and autophagosome clearance in neurons: relationship to autophagic pathology in Alzheimer's disease. J Neurosci 28:6926-6937.

Cruts M, Gijselinck I, van der Zee J, Engelborghs S, Wils H, Pirici D, Rademakers R, Vandenberghe R, Dermaut B, Martin JJ, van Duijn C, Peeters K, Sciot R, Santens P, De Pooter T, Mattheijssens M, Van den Broeck M, Cuijt I, Vennekens K, De Deyn PP, et al. (2006) Null mutations in progranulin cause ubiquitin-positive frontotemporal dementia linked to chromosome 17q21. Nature 442:920-924.

Cuervo AM, Stefanis L, Fredenburg R, Lansbury PT, Sulzer D (2004) Impaired degradation of mutant alpha-synuclein by chaperone-mediated autophagy. Science 305:1292-1295.

Filimonenko M, Stuffers S, Raiborg C, Yamamoto A, Malerød L, Fisher EM, Isaacs A, Brech A, Stenmark H, Simonsen A (2007) Functional multivesicular bodies are required for autophagic clearance of protein aggregates associated with neurodegenerative disease. J Cell Biol 179:485-500.

Hara T, Nakamura K, Matsui M, Yamamoto A, Nakahara Y, SuzukiMigishima R, Yokoyama M, Mishima K, Saito I, Okano H, Mizushima N (2006) Suppression of basal autophagy in neural cells causes neurodegenerative disease in mice. Nature 441:885-889.

Hong M, Zhukareva V, Vogelsberg-Ragaglia V, Wszolek Z, Reed L, Miller BI, Geschwind DH, Bird TD, McKeel D, Goate A, Morris JC, Wilhelmsen KC, Schellenberg GD, Trojanowski JQ, Lee VM (1998) Mutation-specific functional impairments in distinct tau isoforms of hereditary FTDP-17. Science 282:1914-1917.

Hurley JH (2008) ESCRT complexes and the biogenesis of multivesicular bodies. Curr Opin Cell Biol 20:4-11.

Hutton M, Lendon CL, Rizzu P, Baker M, Froelich S, Houlden H, PickeringBrown S, Chakraverty S, Isaacs A, Grover A, Hackett J, Adamson J, Lincoln S, Dickson D, Davies P, Petersen RC, Stevens M, de GraaffE, Wauters E, van Baren J, et al. (1998) Association of missense and 5'-splice-site mutations in tau with the inherited dementia FTDP-17. Nature 393:702-705.
Klionsky DJ, Abeliovich H, Agostinis P, Agrawal DK, Aliev G, Askew DS, Baba M, Baehrecke EH, Bahr BA, Ballabio A, Bamber BA, Bassham DC, Bergamini E, Bi X, Biard-Piechaczyk M, Blum JS, Bredesen DE, Brodsky JL, Brumell JH, Brunk UT, et al. (2008) Guidelines for the use and interpretation of assays for monitoring autophagy in higher eukaryotes. Autophagy 4:151-175.

Komatsu M, Waguri S, Chiba T, Murata S, Iwata J, Tanida I, Ueno T, Koike M, Uchiyama Y, Kominami E, Tanaka K (2006) Loss of autophagy in the central nervous system causes neurodegeneration in mice. Nature 441:880-884.

Kuma A, Hatano M, Matsui M, Yamamoto A, Nakaya H, Yoshimori T, Ohsumi Y, Tokuhisa T, Mizushima N (2004) The role of autophagy during the early neonatal starvation period. Nature 432:1032-1036.

Lee JA, Gao FB (2008) Roles of ESCRT in autophagy-associated neurodegeneration. Autophagy 4:230-232.

Lee JA, Beigneux A, Ahmad ST, Young SG, Gao FB (2007) ESCRT-III dysfunction causes autophagosome accumulation and neurodegeneration. Curr Biol 17:1561-1567.

Mizushima N, Noda T, Yoshimori T, Tanaka Y, Ishii T, George MD, Klionsky DJ, Ohsumi M, Ohsumi Y (1998) A protein conjugation system essential for autophagy. Nature 395:395-398.

Mizushima N, Yamamoto A, Hatano M, Kobayashi Y, Kabeya Y, Suzuki K, Tokuhisa T, Ohsumi Y, Yoshimori T (2001) Dissection of autophagosome formation using Apg5-deficient mouse embryonic stem cells. J Cell Biol 152:657-668.

Mizushima N, Levine B, Cuervo AM, Klionsky DJ (2008) Autophagy fights disease through cellular self-digestion. Nature 451:1069-1075.

Neumann M, Sampathu DM, Kwong LK, Truax AC, Micsenyi MC, Chou TT, Bruce J, Schuck T, Grossman M, Clark CM, McCluskey LF, Miller BL, Masliah E, Mackenzie IR, Feldman H, Feiden W, Kretzschmar HA, Trojanowski JQ, Lee VM (2006) Ubiquitinated TDP-43 in frontotemporal lobar degeneration and amyotrophic lateral sclerosis. Science 314:130-133.

Raiborg C, Stenmark H (2009) The ESCRT machinery in endosomal sorting of ubiquitylated membrane proteins. Nature 458:445-452.

Ravikumar B, Vacher C, Berger Z, Davies JE, Luo S, Oroz LG, Scaravilli F, Easton DF, Duden R, O'Kane CJ, Rubinsztein DC (2004) Inhibition of mTOR induces autophagy and reduces toxicity of polyglutamine expansions in fly and mouse models of Huntington disease. Nat Genet 36:585-595.

Seglen PO, Gordon PB (1982) 3-Methyladenine: specific inhibitor of autophagic/lysosomal protein degradation in isolated rat hepatocytes. Proc Natl Acad Sci U S A 79:1889-1892.

Skibinski G, Parkinson NJ, Brown JM, Chakrabarti L, Lloyd SL, Hummerich H, Nielsen JE, Hodges JR, Spillantini MG, Thusgaard T, Brandner S, Brun A, Rossor MN, Gade A, Johannsen P, Sørensen SA, Gydesen S, Fisher EM, Collinge J (2005) Mutations in the endosomal ESCRTIII-complex subunit CHMP2B in frontotemporal dementia. Nat Genet 37:806-808.

Vossel KA, Miller BL (2008) New approaches to the treatment of frontotemporal lobar degeneration. Curr Opin Neurol 21:708-716.

Watts GD, Wymer J, Kovach MJ, Mehta SG, Mumm S, Darvish D, Pestronk A, Whyte MP, Kimonis VE (2004) Inclusion body myopathy associated with Paget disease of bone and frontotemporal dementia is caused by mutant valosin-containing protein. Nat Genet 36:377-381.

Yue Z, Friedman L, Komatsu M, Tanaka K (2009) The cellular pathways of neuronal autophagy and their implication in neurodegenerative diseases. Biochim Biophys Acta. Advance online publication. Retrieved June 16, 2009. doi:10.1016/j.bbamcr.2009.01.016. 